\title{
Creating readable text: The role of a typographic style in the development and preparation of instructional texts
}

\author{
Neal Kemp and Steve Towers \\ University of Central Queensland
}

\begin{abstract}
As computing technology has changed the way in which teaching materials are prepared, the role of self-instructional texts has attracted greater attention. This paper addresses the role of typographic style in the development of self-instructional text. The authors have identified the difference between the visual appeal of text and the ease with which learners interpret the content. Steps towards developing and implementing a defined typographic style are discussed, including a brief methodology for style management and maintenance. Recommendations are presented about how to maintain style and achieve high quality educational products.
\end{abstract}

Although there have been considerable advances in media technology in recent years, print remains the most widely used medium for providing instructional materials, especially in distance education. The key to providing quality instruction through print lies in the presentation, instructional layout and textual format of the materials, all of which contribute to enhancing the learner's educational experience.

The emergence of "Do-It-Yourself" publishing systems in the mid-1980s offered organisations who were involved in the development of technical documentation the ability to manage and monitor the total preparation of their print materials within their own environments, thereby obviating the necessity, or expense of, outside typesetting facilities.

The enthusiastic proponents of the computer publishing technologies proudly marketed their products on the premise that "typographic quality was inherent in desktop publishing ... otherwise it would be mere word processing!". This misconception was exacerbated by the generalised theory that typographic quality is measured in purely aesthetic terms. 
While in commercial advertising this belief may hold some credibility, the quality of instructional texts is measured in terms of readability and subsequent learner comprehension. Morison (1951) defines typography as that which is "...essentially utilitarian, the enjoyment of patterns being rarely the reader's main aim".

There is no doubt that computer publishing has opened a virtual "Pandora's Box" for those organisations who have traditionally been constrained with inflexible word processing systems. However the extent of typographical options now available on most publishing systems and, more importantly, the effective application of these options in document design, demand a knowledge beyond that previously expected of most word processing operators or typists. In the six years since the introduction of the "Do-It-Yourself" publishing systems, this point has been demonstrated through a proliferation of print material which has favoured the creator's conception of visual appeal over the reader's ability to interpret the communication.

The emergence of computer publishing has provided the tools to enable the developers of technical documentation to shape the presentation of their own material. However, this is often done with little knowledge of the typographical parameters which can affect the readability and subsequent comprehension of their documents. While the "look" of a publication should be of some concern to the publisher, the legibility, readability and instructional presentation of technical documentation will determine its effectiveness.

Anyone who is involved in producing printed materials whether they be technical manuals, advertising brochures, or simple forms is in the business of publishing, and is charged with its inherent responsibilities. As the computer publisher's role is primarily to communicate a message between author and reader, the publisher should be discouraged from experimenting with the reader's ability to work with and comprehend the text. Webster and Larter (1989) contend that the design of the medium should not compete with the message conveyed.

Design is not mere decoration, but part of the business of communication. The concern should not be for the creator's eye for beauty. It should be for those who read a publication and receive the message intended by the author. Good design therefore is a functional process and its success is best measured by its transparency to the reader.

\section{A typographic style}

Among the many sales pitches which surrounded the introduction of the D-I-Y technologies was the inference that anyone could publish. It is 
understandable that, in many cases, the resultant materials were devoid of the essential typographic characteristics which play a major role in determining text readability. The responsibilities, traditionally the domain of the publisher/printer, have been taken over by these newcomers to publishing. These new responsibilities are illustrated in Figure 1.

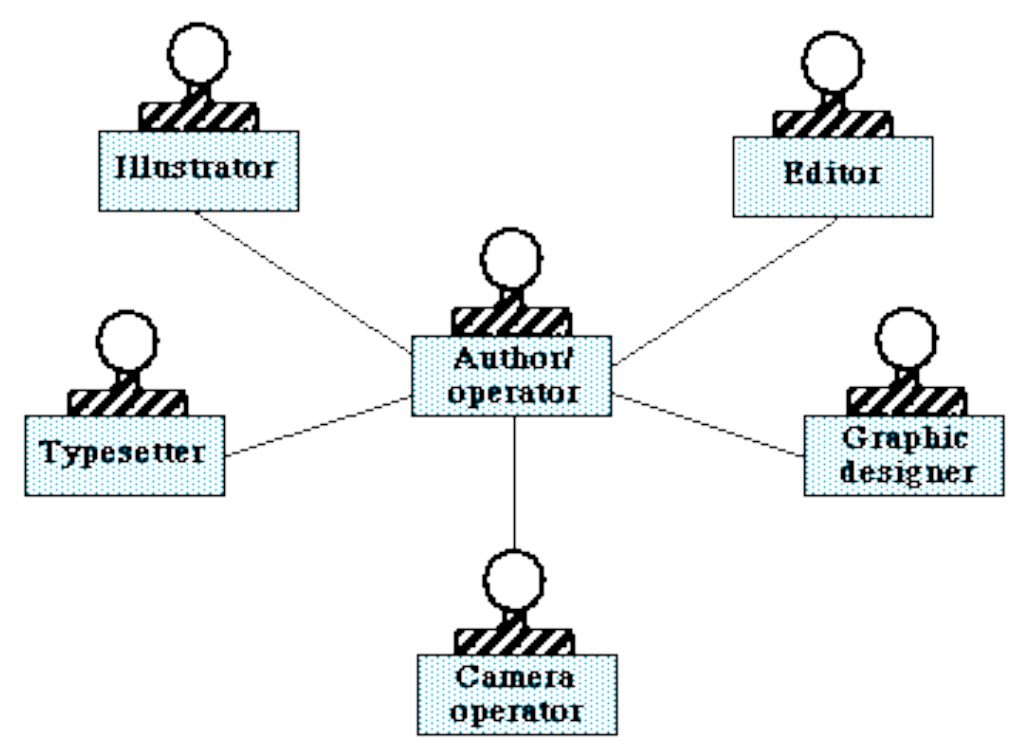

Figure 1

While newly installed computer publishing systems offered an enormous degree of typographic flexibility in comparison to the superseded word processor, it became apparent to many publishers that, if there was no control over the operator's use of these facilities, there would be, ironically, a degradation in the quality of documentation produced.

The requirements for the presentation of technical documentation are not unique However as these materials are intended primarily for selfinstruction, there is a necessity for them to be able to "stand alone" and not require interpretation. In this role, self-instructional texts can be viewed as "tutorials in print" (Rowntree, 1986). Hartley (1985) contends that texts for instruction should provide a consistent frame of reference within which the reader/learner can move about, leave and return without confusion.

In the preparation of technical documentation, it is then perhaps necessary to restrict the choices which can be made by those involved in the development process in regard to the presentation of the text. Such restrictions may best be achieved through the development of a 
typographic standard or style (Dekkers, Kemp and Griffin, 1988) providing guidelines for authors, designers and operators.

A typographic style for the preparation of technical documentation is seen as essential for a variety of reasons:

(a) to facilitate the development of materials by offering authors a variety of instructional features and ideas;

(b) to facilitate the production of materials through its use as a reference manual detailing the typographic format of all components which would typically appear in the document;

(c) to exploit the full functionality of the publishing software and thereby act as a selling tool for the publisher.

\section{Benefits of style}

The development of a typographic style would be greatly beneficial in controlling the quality of text presentation for several reasons:

(a) The typographic parameters which would ensure that documents are prepared in a highly readable format are pre-set and, as such, would not need to be determined prior to the preparation of each document.

(b) It would ensure that production operators have clear guidelines to follow and subsequently produce material in a consistent and efficient manner. This is not to say that operators will not have to make decisions from time to time regarding the format of particular features which are not dealt with in the style, but decision-making at this level should not have a significant impact upon text readability if production procedures are followed. In addition, the adoption of a single style for text formatting means that production time is used more effectively through operators not having to experiment with new styles of layout.

(c) The reader/learner feels comfortable with the instructional material as the format is familiar to them. This consistency in presentation ensures that instructional features of the text are signalled in a consistent manner and that devices used for accessing the structure of the text are common between related documents. The implementation of a consistent presentation style across all subjects in a course should, at least, provide one less barrier to learning for those studying at a distance.

(d) Authors can envisage the presentation of their texts at the writing stage. In the same way that production operators are given clear guidelines for layout, so too are authors presented with a range of carefully designed and tested options for the development of their 
instructional text. In this way a documented style guide also acts as an "ideas" book, presenting alternative strategies to suit particular teaching and learning styles and environments.

(e) The image of the teaching institution is enhanced through consistent presentation of a "corporate" image. With each component of a study package, or a course, carrying a common design theme, there becomes a visually cohesive link which indicates that each is an integral component of a larger family of materials. This "corporate look" can have a major impact upon the marketing of packaged courses between institutions and especially to industry.

\section{Developing style}

It is perhaps presumptuous to assume that the computer publishing operator will possess the appropriate design skills necessary to prepare text which conforms to established typographic principles. This is, however, the expectation of many employers who are subsequently disappointed when their word processing staff are unable to produce the kind of quality documentation they have come to expect from their traditional publisher. These composition skills, which are essential in ensuring the readability and aesthetic appeal of the printed page, typically exist only within the traditional printing and publishing sectors of industry.

It is therefore necessary that a skilled typographic designer be involved in developing a style and moreover that this person, and others who are to be involved, have an intimate knowledge of the functionality of the computer publishing system.

The implementation of a typographic style into an academic publishing environment will not, by itself, result in the production of high quality documentation. In fact, it may take some time for authors to accept the imposition of a set of layout "rules". As a consequence, this publishing style will be closely and constantly scrutinised.

In order to address the issues relating to the acceptance of publishing style by all involved in the publishing process, there are some issues which require consideration at the development stage.

(a) To ensure the production of quality instructional materials that are highly readable and serve to help, rather than hinder, the communication process, it is imperative that reference be made to current and relevant research in the field of instructional typography. There is considerable research (Waller, 1979; Moriarty, 1986; Rehe, 1972; Wheildon, 1986) which targets particular typographic issues in text presentation, while Hartley (1981) adequately summarises into a 
set of guidelines the typographical requirements of sound printed instructional materials.

(b) The development of typographic standards for academic publishing must take into account the specific and unique requirements of each discipline. The typographic presentation of scientific and mathematical notation, for example, demands the integration of a sophisticated mathematics generation utility within the publishing software. Further, it is important that conventions regarding the presentation of scientific notation be adhered to ensure consistency between student reports and study materials.

(c) The development of style should not be solely concerned with satisfying the instructional and typographic needs associated with materials development. Cognisance should also be made of the technical constraints associated with the production and printing processes, for example most publishing systems, desktop output devices, high speed printing and photocopying equipment are designed to handle the ISO A4 paper format. Working to the limits of or outside the technical constraints of production equipment has an exponential effect upon the cost of materials production.

(d) To aid in the development of materials and to expedite the production process it is essential that the typographical style be documented. Documenting the style is also important in respect of quality assurance as decisions for choosing particular typographic parameters can, over time, be tested and improved. Having the ground rules for text presentation clearly documented, with printed examples, should ensure a level of quality through the typesetting process, especially in regard to consistency, a vital component in presenting instructional text (Hartley, 1985). Moreover, a printed style guide can act as an resource book providing authors with alternative strategies for varying the presentation of their instructional texts.

(e) The style development process should include the opportunity for authors to cover discipline-specific nuances and accommodate known learner difficulties. This step allows for information exchange, promotes ownership of the style to all stakeholders and ensures that understanding and rationale for the style is conveyed to all staff.

The implementation of style should ensure conformity in the presentation of instructional materials, which, in terms of the production process, will enable the maintenance of a level of quality. The level of quality is, of course, highly dependent upon the complexity and sophistication of the style which has been developed. Maintaining a level of quality in documentation will be facilitated by the flexibility of the style and its ability to accommodate change as new conventions evolve. 


\section{Style maintenance and management}

The development of a typographic style is a dynamic process, requiring regular review, adjustment and documentation to ensure optimal functionality. While subtle changes to style is a day to day, job by job occurrence, major changes to style may arise from:

(a) Research - relevant research requires regular reviewing to determine new variables effecting readability.

(b) Hardware/ software upgrades / changes - upgrades often present new typographical functionality which allows an expanded range of features that may be included in the style, whereas changes may add, limit or subtract features.

(c) Evaluation - evaluation of the effectiveness of the instructional materials may require modification to their layout.

(d) Feedback - instructional design staff may identify common problems that may be accommodated through a change in style.

To accommodate a dynamic style, commitment to on-going training is essential. The training plan should include all stakeholders, that is administrators, authors, instructional designers and production staff. While the focus, duration and regularity for the various stakeholders will differ greatly, their inclusion in a training program is never-the-less important.

Interaction among stakeholders should be fostered and encouraged through regular meetings which should be integral to the production process. Dissatisfaction for style and production procedures has often arisen through lack of understanding and ignorance of roles and capabilities.

\section{Conclusion}

Print remains the most popular and cost effective medium for the mass dissemination of materials for instruction. Computer publishing technology offers tremendous potential and instructional flexibility to produce low cost, quality, print-based materials. However, to positively harness the full potential of the computer publishing technologies, managers must approach implementation systematically.

Central to any implementation strategy is the development, documentation and maintenance of a typographic style to maximise the instructional quality of learning materials and to provide authors and designers with a consistent and comprehensive range of typographical tools for teaching in text. Style development should be grounded in 
research and facilitated by an expert in typographic design with relevant publishing experience. The style is useful to operators and authors by offering a range of typographical options within acceptable readability limits based on appropriate research and experience. Style should be viewed as a dynamic process that requires regular training for all staff involved in the publishing process to ensure the maintenance of high quality publications.

\section{References}

Commonwealth of Australia. (1978). Style manual for authors, editors and printers. Canberra: Australian Government Publishing Service.

Dekkers, J., Griffin, H and Kemp, N. (1988). Style guide. Rockhampton: Capricornia Institute.

Hartley, J. (1981). Eighty ways of improving instructional text. IEEE Transactions on Professional Communication, PC-24, 1, March.

Hartley, J. (1985). Designing Instructional Text, (2nd edn). London: Kogan Page.

Moriarty, S. (1986). A search for the optimum line length. Journalism Quarterly, 63, 2, 337-340.

Moriarty, S. (1986). Line lengths and starch scores. Visible Language, 20(4), Autumn, 448-455.

Morison, S. (1951). First principles of typography. London: Cambridge University Press.

Rehe, R. (1972). Proposals for a functional typography. Bloomington, Indiana: Indiana University.

Rowntree, D. (1986). Teaching through self-instruction: A practical handbook for course developers. London: Kogan Page.

Waller, R. (1979). Typographic access structures for educational texts. In Kolers, P., Wrolstad, M. and Bouman, P. (eds.), Processing Visible Language. New York: Plenum.

Wheildon, C. (1986). Communicating or just making pretty shapes. Sydney: Newspaper Advertising Bureau of Australia

Wheildon, C., Webster, T. and Larter, B. (1989). Design and layout in computer publishing. Belrose: Webster and Associates.

Authors: Neale Kemp is Manager, Production and Systems, Distance Education Centre, University of Central Queensland and Steve Towers is the Associate Director, Queensland Open Learning Network. Their address for correspondence is Division of Distance and Continuing Education, University of Central Queensland, Rockhampton Campus, Rockhampton, Queensland 4702.

Please cite as: Kemp, N. and Towers, S. (1992). Creating readable text: The role of a typographic style in the development and preparation of instructional texts. Australian Journal of Educational Technology, 8(1), 27-34. http: / / www.ascilite.org.au / ajet/ajet8/ kemp.html 